\title{
Vibration Sideband Modulations and Harmonics Separation of a Planetary Helicopter Gearbox with Two Different Configurations
}

\author{
Nader Sawalhi \\ Mechanical Engineering Department, Prince Mohammad Bin Fahd University, AL-Khobar, Saudi Arabia \\ Correspondence should be addressed to Nader Sawalhi; nadersaw@hotmail.com
}

Received 20 June 2016; Revised 19 September 2016; Accepted 11 October 2016

Academic Editor: Kim M. Liew

Copyright @ 2016 Nader Sawalhi. This is an open access article distributed under the Creative Commons Attribution License, which permits unrestricted use, distribution, and reproduction in any medium, provided the original work is properly cited.

This paper examines the spectrum and cepstrum content of vibration signals taken from a helicopter gearbox with two different configurations ( 3 and 4 planets). It presents a signal processing algorithm to separate synchronous and nonsynchronous components for complete shafts' harmonic extraction and removal. The spectrum and cepstrum of the vibration signal for two configurations are firstly analyzed and discussed. The effect of changing the number of planets on the fundamental gear mesh frequency (epicyclic mesh frequency) and its sidebands is discussed. The paper explains the differences between the two configurations and discusses, in particular, the asymmetry of the modulation sidebands about the epicyclic mesh frequency in the 4 planets arrangement. Finally a separation algorithm, which is based on resampling the order-tracked signal to have an integer number of samples per revolution for a specific shaft, is proposed for a complete removal of the shafts harmonics. The results obtained from the presented separation algorithms are compared to other separation schemes such as discrete random separation (DRS) and time synchronous averaging (TSA) with clear improvements and better results.

\section{Introduction}

Vibration signals originating from a helicopter transmission gearbox represent a rich source for monitoring its health. Many failures that occur in rotating components such as gears and bearings often show their signature in the vibration signal and can be well detected at early stages. Monitoring these vibrations often requires an extensive interpretation by a trained diagnostician, due to the complexity of such systems [1]. A major part of this involves the correct understanding and identification of the frequency content of the vibration signal. Understanding the frequency content of the signal and the different families of harmonics and sidebands would enable a correct analysis of the health of the machine.

Signals are mixtures of different sources. For successful handling and interpretation of signals, analysts often need to separate these different sources and process them separately. One of the most successful ways of interpreting signals is the use of Fast Fourier Transformation (FFT), which transforms the signal from the time domain into the frequency domain by using sines and cosines as base functions for the signal decomposition. FFT requires the transformed signal to be stationary; that is, it has some statistical parameters which do not change with time. For nonstationary signals (have time dependent statistics), the use of time-frequency presentation such as the spectrogram (short time-frequency analysis), the wavelets, the winger vile transform, and so forth is commonly used. Stationary signals are mainly composed of deterministic (discrete) components and random components. Random components contain all nonstationary signals in addition to any nondeterministic part. Deterministic components are those which can be expressed as a series of discrete sinusoidal signals (thus they are predictable and periodic). Deterministic component can be interchangeably referred to as discrete signals. They generally fall into two main categories:

(i) Periodic (cyclic): they are composed of sinusoids whose frequencies are all integer multiples of some fundamental frequency like the shaft speed in rotating 


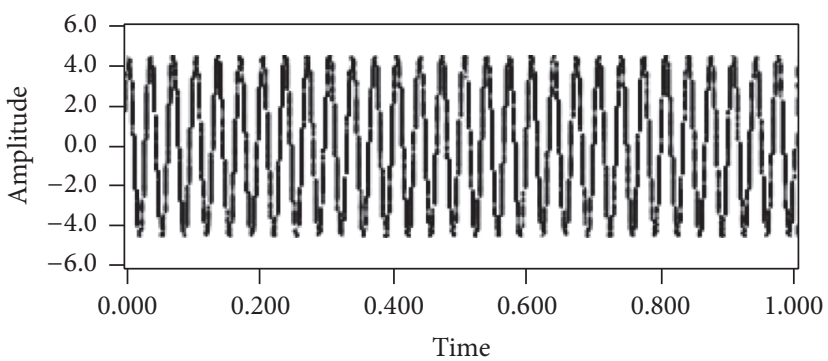

(a) Carrier signal

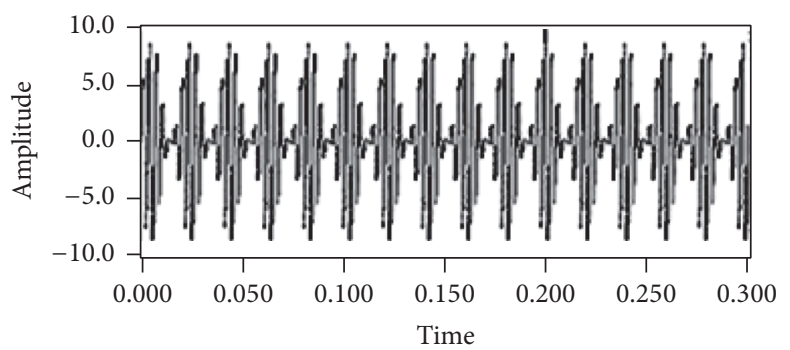

(c) Modulated signal in time

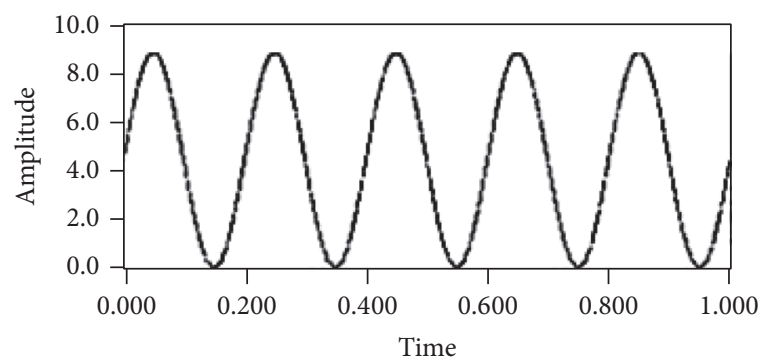

(b) Modulating signal

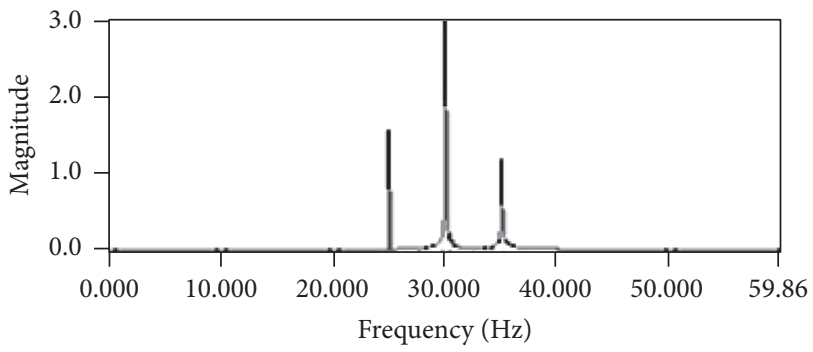

(d) Modulated signal in frequency domain

FIgURE 1: An example of amplitude modulation and sidebands.

machinery; The multiples of the fundamental frequencies are known as harmonics, with the fundamental being the first harmonic; periodic components can also manifest themselves as sidebands around a carrier frequency in the case of a modulated signal (e.g., a gearbox signal where the shaft speed (low frequency) modulates the gear mesh frequency (high frequency))

(ii) Quasi-periodic: they have at least two frequency components that are not rationally related and thus never repeated themselves exactly

Signal modulation or distortion occurs when the amplitude, frequency, or phase of a waveform is altered by the introduction of another physically related periodic signal or disturbance. The high frequency signal is known as the "carrier." The spectrum of the combined signal exhibits a discrete and dominant frequency (carrier) bounded by "sidebands" or peaks (modulator) spaced on either side of the carrier at the modulation frequency. Figure 1 illustrates the amplitude modulation. Figure 1(a) is the carrier signal, a pure sine wave with $30 \mathrm{~Hz}$ frequency in the time domain. Figure 1(b) displays the modulating signal, a pure sine wave with $5 \mathrm{~Hz}$ frequency. Figure 1 (c) shows the modulated signal in the time domain. Figure $1(\mathrm{~d})$ is the modulated signal in the frequency domain. The two sidebands can be identified easily.

For example, gear mesh frequency is a fixed multiple of rotating speed and is displayed as a distinct peak in the spectrum. If there are defects associated with the gearing (physically related) such as gear eccentricity or excessive wear that generate a synchronous force variation, a disturbance will accompany the gear mesh frequency. The high frequency gear mesh is the carrier. The low frequency gear defects will appear as "sidebands" on either side of the gear mesh peak.
The spectral distance from the peak to the side band peak is equal to frequency modulation or the rotating speed of the defective gear. Multiple gear defects are reflected as an extra set of sidebands.

Helicopter transmission gearboxes have been investigated in detail to understand the patterns of baseline frequencies and failure indicators that can be detected by monitoring vibrations $[2,3]$. Those efforts resulted in a better understanding of the way in which different signals manifest themselves and mix with others and led to the development of a variety of diagnostic and prognostic techniques [4-6].

In general, interpreting the vibration signal transmitted through those gearboxes often requires more than the traditional inspection of the time signal and/or its frequency content. This is due mainly to the existence of a large number of rotating components, all of which contribute and mix in different ways [7], making it very hard to track changes in a certain component. In a planetary gearbox, it is quite important to investigate the different setups and determine the expected frequencies in the spectrum. Two examples are presented here and discussed for the Bell 206 helicopter gearbox.

Another important aspect which is presented in this paper is the separation of the gear mesh frequencies and shafts harmonics form the spectrum, using the information from only one tachometer, to enable further processing of the signal to detect gears and bearings faults. The results obtained from this analysis are compared to time synchronous averaging (TSA) processing and discrete random separation (DRS) [8].

When using TSA, the signal is resampled into the angular domain (rather than the temporal domain) to have the same number of samples for each shaft revolution. This removes any speed fluctuation from the signal. The shaft harmonics, 
now called orders, become locked to the shaft rotation and appear as discrete components in the frequency domain (with no order tracking, the higher harmonics usually smear and become broad). The ensemble average for all the rotations is calculated to give the so-called "synchronous average," which represents one shaft rotation and captures the deterministic part of the signal. If the synchronous average is subtracted from the signal, the result will be a residual, which contains noise, nonstationary signals, and any nondeterministic signal. TSA requires the presence of a tachometer signal for the order tracking stage.

DRS works well in the cases of slight (small) speed variation but requires a number of parameters to select and leaves notches in the signal at the locations of the discrete components. A linear transfer function (similar to the H1 transfer function estimate in modal analysis) is generated between the signal and a delayed version of it (using FFT). This gives a value of 1 for the discrete frequency bins and 0 elsewhere. This filter (amplitude of the transfer function) is then used to filter the signal and separate out the discrete components. The filtration is all based on the efficient FFT methods and thus the processing is computationally fast. The two main parameters required are the filter length and the amount of delay to capture the deterministic signals. There are a number of recommendations and a visual examination is usually required to set up these values to give the required separation. This method blindly removes all the discrete components from the whole frequency bandwidth and leaves notches in the spectrum at the location of these frequencies.

This paper is organized as follows. In Section 2, Bell 206 transmission setup and expected frequencies are presented. Section 3 compares the fundamental epicyclic mesh frequency and sidebands between the three- and four-planet arrangements. Section 4 presents the algorithm for the removal of synchronous shaft signals through resampling and signal truncation. The paper is concluded in Section 5.

\section{Bell 206 Transmission Setup and Expected Frequencies}

Although it is not necessary to be an expert in gear system design, it is essential to fully understand the power flow through the gears, the rate of rotation of each component as a function of input shaft speed, the number of teeth on each gear, and the placement/identification of bearings. Due to the variety of components, gear system frequencies typically populate a wide portion of the spectrum from less than shaft speed (tooth repeat frequencies) to multiples of gear mesh frequency.

Gear mesh frequency is defined as the number of teeth on the gear multiplied by the rotational speed of the shaft. Gear mesh is the key parameter to monitor, as any anomaly in the transfer of power through the gears will be reflected at this frequency.

Figure 2 illustrates a schematic presentation of the Bell 206 planetary gearbox with three-planet arrangement. Three accelerometers are fitted on the ring gear (front, rear, and left hand side). The different parameters of the gear system as well

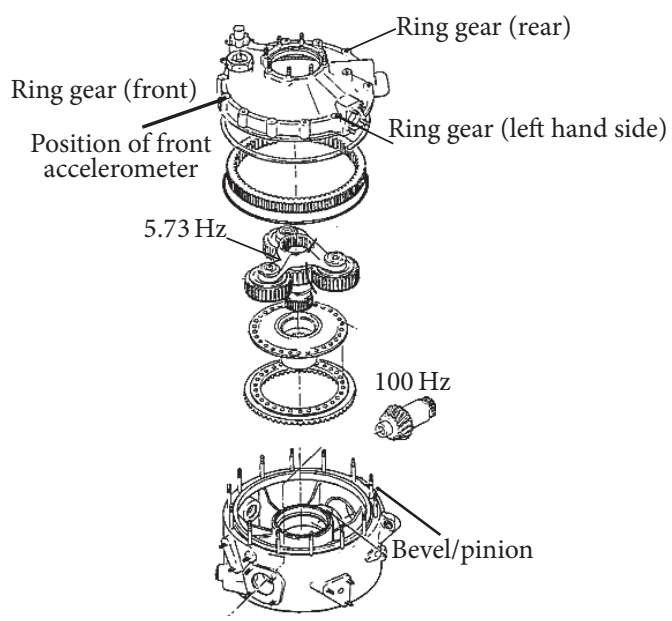

Figure 2: Bell 206 transmission with 3 planets.

TABLE 1: Bell 206 gears, speed, and data acquisition parameters.

\begin{tabular}{|c|c|c|}
\hline Parameter & Value & Reduction ratios \\
\hline Shaft input speed (Hz) & 100 & \multirow{4}{*}{$\begin{array}{l}\text { First-stage reduction } \\
\text { ratio: } \\
3.73: 1(71 / 19)\end{array}$} \\
\hline Number of stages & 2 & \\
\hline $\begin{array}{l}\text { Number of teeth of the } \\
\text { bevel pinion }\end{array}$ & 19 & \\
\hline $\begin{array}{l}\text { Number of teeth of the } \\
\text { bevel gear }\end{array}$ & 71 & \\
\hline $\begin{array}{l}\text { Number of teeth of the sun } \\
\text { gear }\end{array}$ & 27 & \multirow{2}{*}{$\begin{array}{l}\text { Second-stage } \\
\text { reduction ratio: } \\
4.67: 1(99 / 27+1)\end{array}$} \\
\hline $\begin{array}{l}\text { Number of teeth of the } \\
\text { planet gear }\end{array}$ & 35 & \\
\hline \multirow{2}{*}{$\begin{array}{l}\text { Number of teeth of the ring } \\
\text { gear }\end{array}$} & 99 & \\
\hline & & $\begin{array}{l}\text { Overall reduction } \\
\text { ratio: } \\
\text { (1st stage times 2nd } \\
\text { stage) } \\
17.45: 1\end{array}$ \\
\hline $\begin{array}{l}\text { Carrier }(\operatorname{arm}) \text { output speed } \\
(\mathrm{Hz})\end{array}$ & 5.73 & \\
\hline Sampling frequency $(\mathrm{Hz})$ & 51200 & \\
\hline Length of records & 30 seconds & \\
\hline
\end{tabular}

as the data acquisition parameters are presented in Table 1. The data from the front accelerometer was used in examining the vibration content and observes variation between the three- and four-planet arrangements.

Shaft speeds and gear mesh frequencies and their calculations for the 3-and 4-planet arrangements are presented in Table 2. Both have the same frequencies except for the planet pass frequency (number of planets times the carrier speed). References [6, 7] can be consulted for detailed calculations of the kinematics of planetary gearboxes (both gears and bearings). 
TABLE 2: Shaft and gear mesh frequencies.

\begin{tabular}{|c|c|c|c|c|}
\hline & Frequency of interest $(\mathrm{Hz})$ & $\begin{array}{l}\text { Three } \\
\text { planets }\end{array}$ & $\begin{array}{c}\text { Four } \\
\text { planets }\end{array}$ & Relationships and calculations \\
\hline \multirow{3}{*}{ Stage 1} & Input shaft frequency & 100.00 & 100.00 & \multirow[b]{2}{*}{$\begin{array}{l}\text { Number of teeth of the bevel pinion times input shaft } \\
\text { frequency }\end{array}$} \\
\hline & Pinion/bevel mesh frequency & 1900.00 & 1900.00 & \\
\hline & Bevel gear shaft speed & 26.76 & 26.76 & Input shaft frequency/1st-stage reduction ratio \\
\hline \multirow{3}{*}{ Stage 2} & $\begin{array}{l}\text { Carrier frequency (output arm } \\
\text { speed) }\end{array}$ & 5.73 & 5.73 & Input shaft frequency/overall reduction ratio \\
\hline & Epicyclic mesh frequency & 567.71 & 567.71 & Number of teeth of the ring gear times carrier frequency \\
\hline & Planet pass frequency & 17.20 & 22.94 & Number of planets times the carrier frequency \\
\hline
\end{tabular}

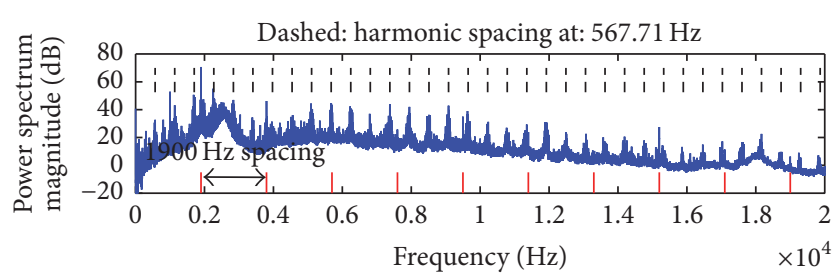

(a)

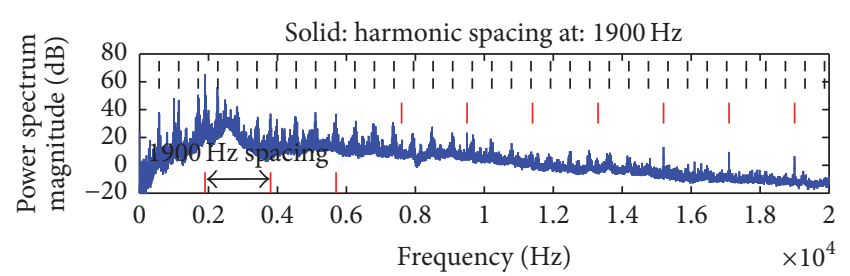

(b)

FIgURE 3: (a) Power spectrum density (PSD) of 3-planet arrangement. (b) Power spectrum density of 4-planet arrangement.

\section{Fundamental Epicyclic Mesh Frequency and Sidebands Comparisons between the Three- and Four-Planet Arrangements}

High quality gears are designed to transfer power by a combined sliding and rolling motion from one gear to the next as smoothly, quietly, and efficiently as possible. This statement means that, starting from the proper metal, the gear teeth are cut to precise dimensions about the geometric center. The tooth surfaces are ground smooth and any imperfections are removed. Each gear is properly fitted to a straight shaft to eliminate eccentricity. The shafts are spaced to optimize the tooth engagement. Any errors in gear manufacture or assembly and/or deterioration will result in a disturbance at gear mesh frequency with adjacent sidebands reflecting once per revolution modulation caused by pitch abnormalities in one or both gears. Since perfection is an asymptotic endeavor, gear systems always display some gear mesh activity. Thus, the presence of the frequency is acceptable and should be apparent. The amplitude increases as the load on the gearing increases. Hence, unless the load is steady, monitoring the amplitude can be a bit tricky. However, an unexplained and significant increase in amplitude at gear mesh frequency is cause of concern.

A large increase in sideband amplitude suggests that something is changing in the geometry and is also cause of concern. The relative amplitude of sideband to gear mesh peak is a good parameter to watch. Scrutinize the waveform for periodic impacts that relate to rotational speed of the gears. Since bearing failure will permit unexpected shaft displacement thereby upsetting gear engagement, always be on the alert for bad bearings. Often, bearing failure precedes gear damage. Increased impacting from deterioration in the transfer of power may excite gear natural frequencies.

A comparison between the three planets and four planets frequency contents from the front accelerometer is shown in Figure 3. The pinion/bevel mesh frequency harmonics are denoted by the dotted cursor, while epicyclic mesh frequency harmonics are indicated using solid line (these can be seen at the $x$-axis). The general overall spectra for both configurations are the same, but this is investigated in further zoom-in analysis around the epicyclic mesh frequency as shown in Figure 4.

The number of planets in the system affects the planet pass frequency (number of planets times the carrier speed) and may also cause an asymmetry of the modulation sidebands about the epicyclic mesh frequency (carrier speed times the number of teeth on the ring gear). In some cases, this may also cause complete suppression of the component at the epicyclic mesh frequency [5]. Thus, although $567.71 \mathrm{~Hz}$ (99 times 5.73 [99x]) is the listed epicyclic mesh frequency in Table 2, it is not necessary that this will be the dominant harmonic. For evenly spaced planets, the epicyclic mesh frequency will be the dominant only if the number of teeth on the ring gear is an integer multiple of the number of planets (ratio of number of teeth on the ring/number of planets is integer) as discussed and detailed in reference [5]. This implies that the energy of the signal will be concentrated at integer multiples of the planets and this what will determine the suppression/nonsuppression of certain sidebands. For Bell206-B3, this ratio is integer (99/3), and the epicyclic mesh frequency and its harmonics are not expected to be suppressed; thus, the epicyclic mesh frequency will have the highest amplitude with sidebands around it. For the Bell-206-B4, 


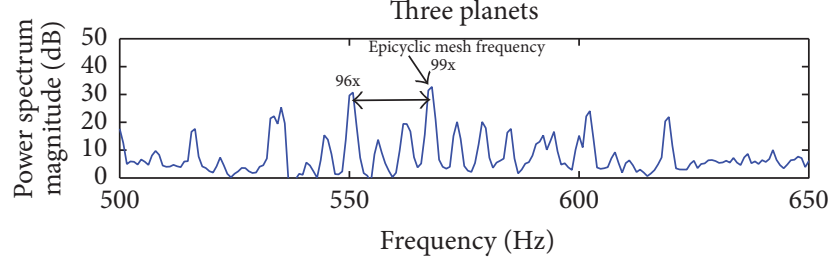

(a)

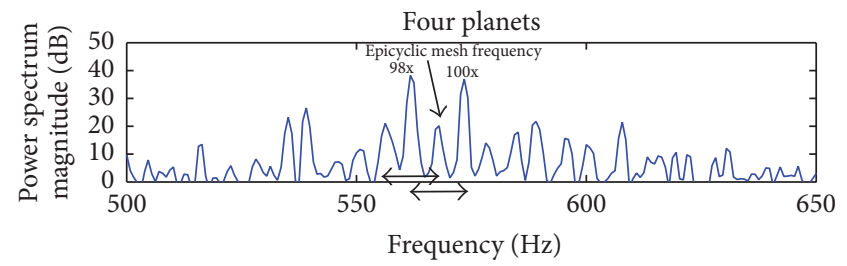

(b)

FIGURE 4: Zoom in PSD showing the epicyclic mesh frequency and its side bands: (a) 3 planets and (b) 4 planets (epicyclic mesh suppressed).

TABLE 3: Bell-206-B4 gearbox modulation sidebands (assuming planets are evenly spaced).

\begin{tabular}{lcc}
\hline Sidebands & $m=1$ & $\left(N_{\text {ring }}+\right.$ Sideband $) / N_{\text {planet }}$ \\
\hline-5 & 0 & $(99-5) / 4(\mathrm{NI})$ \\
-4 & 0 & $(99-4) / 4(\mathrm{NI})$ \\
$-\mathbf{3}(\mathbf{5 5 0 . 5 2} \mathbf{H z})$ & $\mathbf{1}$ & $(\mathbf{9 9 - 3 ) / 4 ( I )}$ \\
-2 & 0 & $(99-2) / 4(\mathrm{NI})$ \\
$-1(561.97 \mathrm{~Hz})$ & 0 & $(99-1) / 4(\mathrm{NI})$ \\
$0(567.71 \mathrm{~Hz})$ & 0 & $(99-0) / 4(\mathrm{NI})$ \\
$\mathbf{1}(\mathbf{5 7 3 . 7 4} \mathbf{H z})$ & $\mathbf{1}$ & $(\mathbf{9 9}+\mathbf{1}) / \mathbf{4}(\mathrm{I})$ \\
2 & 0 & $(99+2) / 4(\mathrm{I})$ \\
3 & 0 & $(99+3) / 4(\mathrm{NI})$ \\
4 & 0 & $(99+4) / 4(\mathrm{NI})$ \\
$\mathbf{5}(\mathbf{5 9 6 . 3 6} \mathbf{H z})$ & $\mathbf{1}$ & $\mathbf{9 9 + 5 ) / 4 ( I )}$
\end{tabular}

0 : suppressed. 1: dominance: nonzero vibration. $N_{\text {ring }}$ : number of teeth on the ring gear ( 99 teeth). $N_{\text {planet }}$ : number of planetary gears (4 planets). I: integer. NI: noninteger.

this ratio (99/4) is not integer and it is expected then that most of the harmonics of the epicyclic mesh frequency will be suppressed (other sidebands will appear strongly). A prediction based on McFadden and Smith's paper [2], for the Bell-206-B4 gearbox, assuming that the planets are evenly spaced is given in Table 3 for the first harmonic of the epicyclic mesh frequency $(m=1)$. Table 3 suggests that the epicyclic mesh frequency will be suppressed and that the sidebands $(550.52,573.74$, and $596.36 \mathrm{~Hz})$ will appear strongly in the spectrum. Note that only the vibration at the first upper sideband and every forth sideband around is nonzero.

To see if this agrees with the actual frequency content of the signal, a zoom-in around the fundamental epicyclic for the 3- and 4-planet arrangements is plotted and presented in Figure 4. It is noticed that, in the 3-planet arrangement, there is no suppression for the epicyclic mesh frequency as predicted earlier (ring number of teeth is exact multiple of the number of teeth on the planet).

For the four-planet arrangements, there is suppression of the epicyclic mesh frequency. The suppression of the epicyclic mesh frequency is quite obvious; however, the sidebands with the highest $\mathrm{dB}$ values (lower and upper sidebands, with a maximum $\mathrm{dB}$ at the 1st lower sideband at $562 \mathrm{~Hz}$ ) do not agree with the predictions of Table 3 (third sideband to the left and 1st upper sideband). This could be explained by the fact that the derivation of Table 3 is based on the assumption that the planets are evenly spaced, which could not be the case for the Bell-206-B4. In reference [6], a system completely similar to Bell-206-B4, referred to as OH-58 C, is discussed. It is stated that the system contains four nonuniformly spaced planet gears and that the planet gears come in pairs spaced 180 degrees apart with the angle between the pairs other than 90 degrees. This explains the observation of Figure 4, as it is the vibration of the pairs (two planet gear sets) rather than the individual planets (4 planets) that determine the nonzero vibration. In this case the highest amplitude is expected to be for integer multiples of 2 rather than 4 and this explains the lower sideband (98 times the carrier speed (a multiple integer of 2 not 4)) and the upper sideband (100 times the carrier speed (multiple integer of both 2 and 4 )).

A further useful summary of these discussed observations can be seen clearly through inspecting the real cepstrum of the two signals as shown in Figure 5. The cepstrum [9] is the inverse Fourier transform of the log spectrum; thus, it has the units of time (Figure 5 shows the time in samples). Cepstrum is a very useful tool to characterize families of sidebands and modulation "in a clear-easy" to interpret format as these become concentrated in harmonics. The dominance of the planet pass frequency in the 3 planets configuration is obvious from Figure 5(a), while a half planet pass presence is present in the 4 planets, which clearly agrees with observation and discussions deduced from the spectrum analysis.

\section{Extraction and Removal of Synchronous Shaft Signals through Resampling and Signal Truncation}

In order to extract and then remove the harmonics related to each shaft (input, intermediate, and output shafts), three tachometers are required if the shafts are independent, for example, aero engines. In such cases, the angular resampling process should be repeated to allow the order tracking of the speeds of the three shafts, and their harmonics could be removed by subtracting the synchronous average after each resampling step. Order tracking (angular resampling) involves resampling the signal at equal intervals of shaft rotation rather than equal time intervals. This removes any speed fluctuation so that the harmonics of the shaft are genuinely discrete frequencies. This enables their removal by performing synchronous averaging of the order-tracked signal and subtracting it from the latter. Order tracking can be performed by phase demodulating a tacho or shaft encoder 


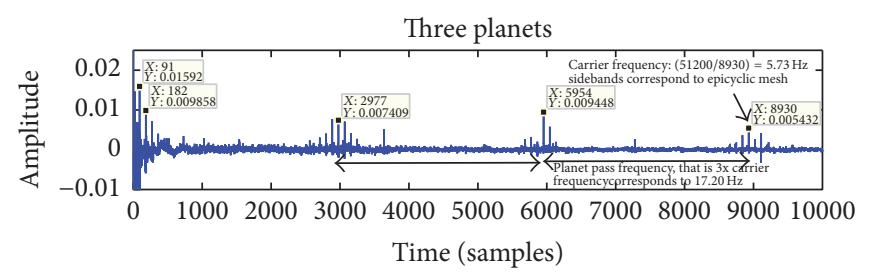

(a)

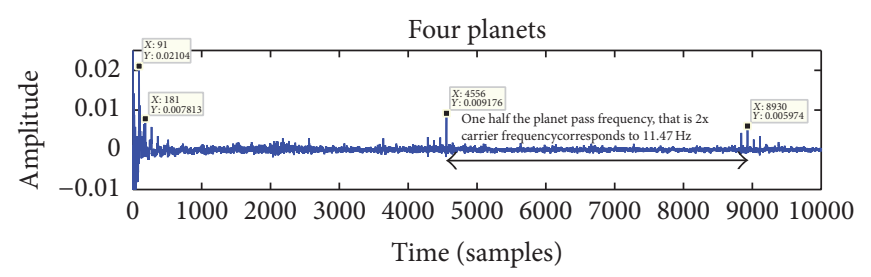

(b)

FIgURE 5: Real cepstrum for the three- and four-planet arrangements: (a) 3 planets and (b) 4 planets.

signal and using the mapping between the shaft angle and time to make interpolations in either direction. The process includes the use of cubic spline interpolation of the vibration signal to calculate the values at the required sample points, based on the tachometer signal.

In gearboxes, only one speed reference signal is provided, which is usually sufficient for removing the harmonics of the three related shafts.

A synchronous averaging separation algorithm [10], which is based on signal resampling, is utilized here for the extraction of the shafts' synchronous signals and separating other nonsynchronous signals. In this sense, there is no difference between the 3-and 4-planet setups as the processing algorithm will remove families of harmonics regardless of what the level of the sidebands is.

This algorithm works by resampling the order-tracked signal to obtain an integer number of samples per revolution for a specific shaft. This enables the removal of the shaft harmonics without much disruption of the vibration signal. As there are only three main shafts in the gearbox arrangement (input, intermediate, and output shafts), this will provide a quick yet efficient way of removing all the harmonics of the three shafts (complete removal of shaft/gear related components). The removal of the harmonics of a specific shaft can be achieved by one of two methods. The first is by finding the synchronous average and subtracting it (repeated periodically) from the signal. The second is by truncating the signal to an integer number of revolutions (preferably a power of 2) as described next and setting the lines corresponding to the harmonics of that shaft (after FFT analysis) to the mean value of the adjacent frequencies. To avoid treating the negative frequency components, it is recommended to set them to zero after the FFT step and double the positive frequency components and then take the real part of the resulting analytic signal in the time domain. Both methods give the same results but with less processing time when using the synchronous averaging method.

The steps included in the algorithm are listed below and presented schematically in Figure 6:

(1) Order track the raw signal based on the input shaft tacho. Ensure that the number of samples per revolution of the input shaft (SPR $i$ ) is an integer number (next power of 2 of the nominal numbers of samples between two shaft rotations).

(2) Find an integer number of periods $(p)$ to truncate the signal to.

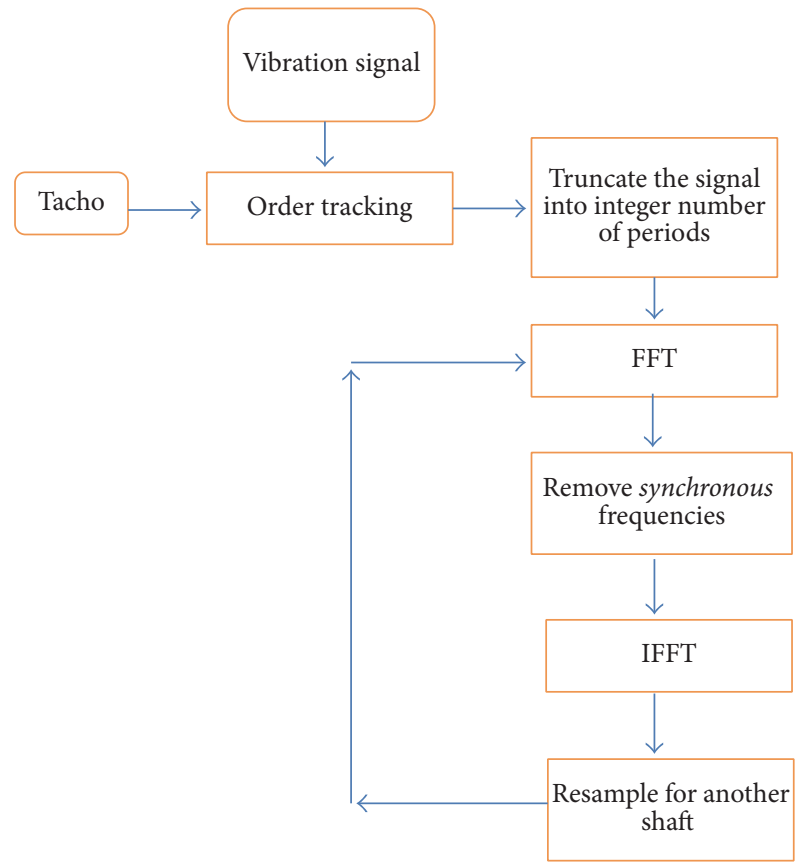

FIGURE 6: Signal separation algorithm.

(3) Truncate the signal to (Nfft) samples (preferably a power of 2), which is equivalent to $p$ periods of the input shaft (i.e., $\mathrm{Nfft}=p \times \mathrm{SPR}_{-} i$ ).

(4) Take the Fast Fourier Transform (FFT) of the truncated signal.

(5) Remove synchronous frequencies related to the input shaft by setting the frequency lines of $(n p+1)$ of the fundamental frequency and its harmonics (up to SPR $i-1)$ to the mean value of its adjacent frequencies. The result to this stage is illustrated in Figure 7 (further illustrated through Figure 8). Figures 7 and 8 show the removal of the harmonics of the input shaft (i.e., the $100 \mathrm{~Hz}$ harmonic). In particular, the pinion/bevel mesh frequency at $1900 \mathrm{~Hz}$ (19x 100), which is shown in dark color in both figures, is completely removed. To avoid treating the positive and negative frequency, it is advised to set the negative frequencies to zeros.

(6) Perform an Inverse Fast Fourier Transform (IFFT) on the resulting frequency content obtained in step (5) 


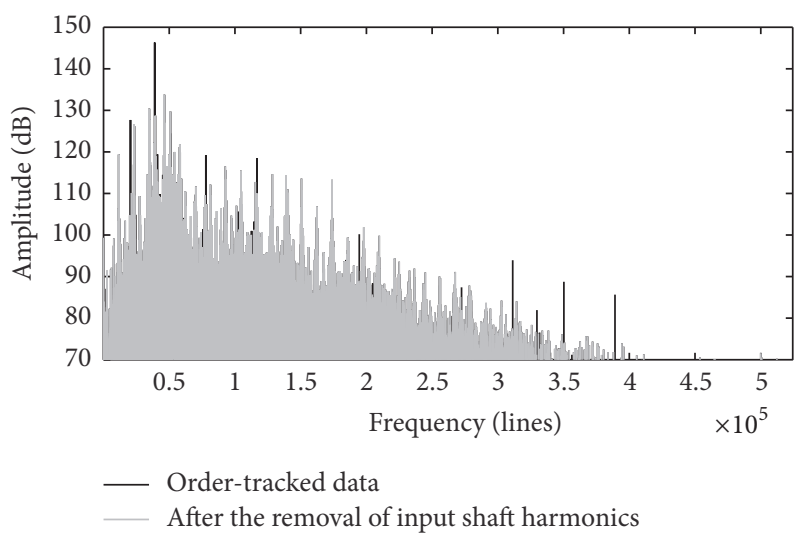

FIGURE 7: Removing the harmonics of the input shaft speed: dark: before the removal; light: after the removal.

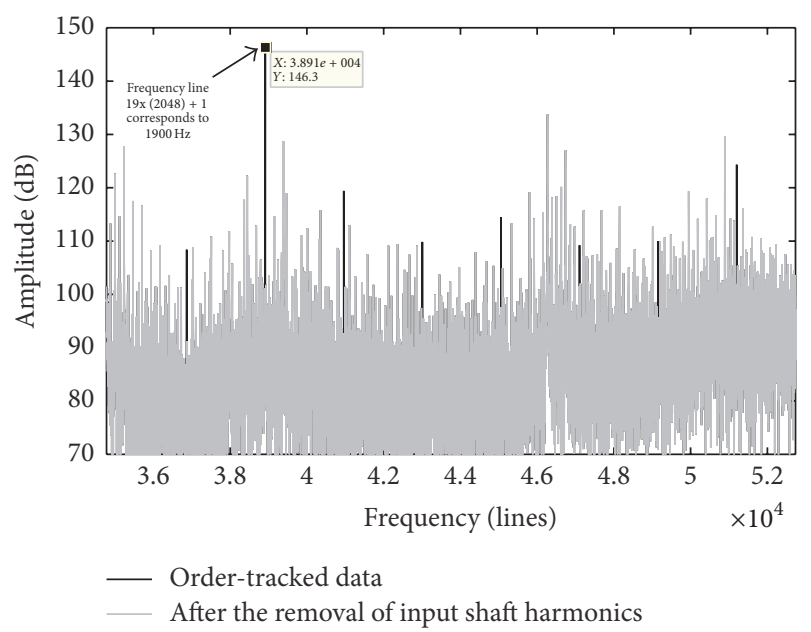

FIGURE 8: Zooming-in around the pinion mesh frequency: dark: before the removal; light: after the removal.

back to the time domain. If the negative frequencies were set to zero, the real part of the signal should be obtained.

(7) Resample so that there is an integer number of samples (power of 2) for each revolution of the intermediate shaft (this can be achieved by working out the gear ratio and using that to resample the signal to give an integer number of samples per revolution of the intermediate shaft).

(8) Repeat steps (4) to (6) to remove the harmonics related to the intermediate shaft.

(9) Resample the obtained signal so that there are an integer number of samples (power of 2 ) for each revolution of the output shaft (use gear ratio to resample the signal so that an integer number of samples per revolution of the output shaft are achieved).

(10) Repeat steps (4) to (6) to remove the harmonics related to the intermediate shaft.

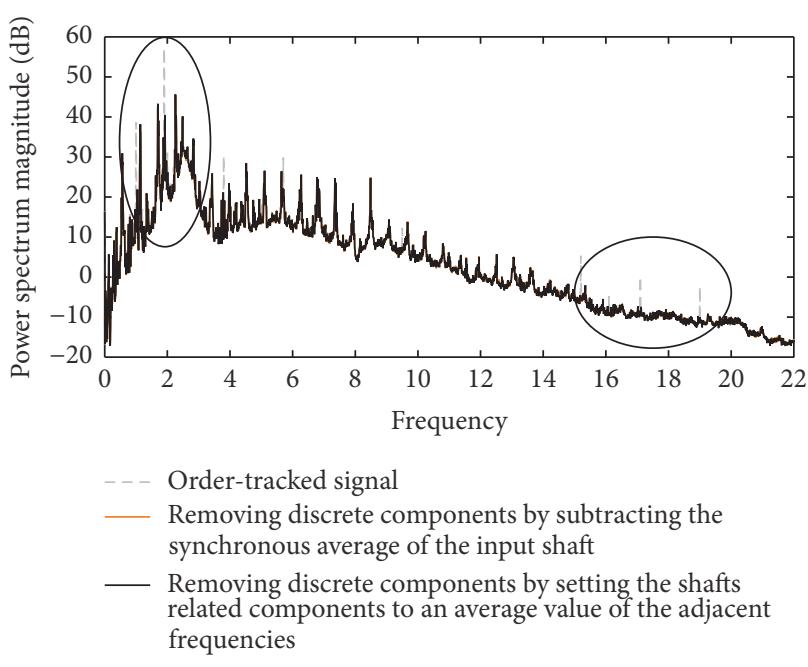

Figure 9: Power spectrum showing the effectiveness of the discrete removal algorithm.

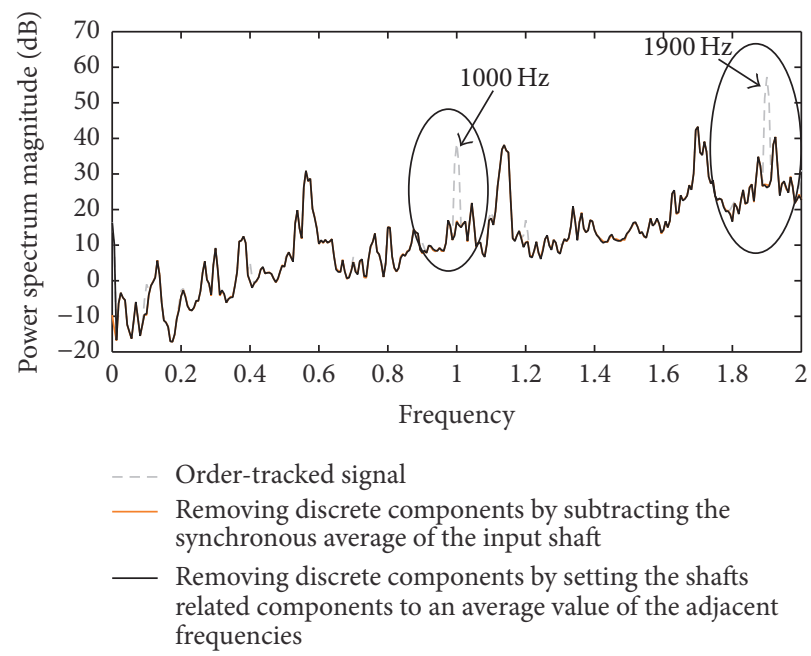

Figure 10: Zoom-in $(0-2000 \mathrm{~Hz})$ : light: signal before processing; dark: signals obtained through different approaches (synchronous averaging subtraction and harmonic removals in the frequency domain).

The output until step number (6) is equivalent to removing the synchronous average after order tracking the signal with respect to the input shaft (i.e., only one set of harmonics, related to the input shaft speed $(100 \mathrm{~Hz})$, is removed at this stage). This is shown (by inspecting the power spectrum) in Figures 8 (whole frequency range) and $9(0-2000 \mathrm{~Hz}$ ). Note how removing the input shaft speed harmonics by subtracting the synchronous average of the order-tracked signal gives a similar result to the one obtained when processing the signal through steps (1)-(7).

Note the circled parts of Figures 9 and 10, which correspond to the harmonics of the input shaft. Note that the epicyclic mesh frequency, its sidebands, and harmonics are still present at this stage and further processing is needed to remove them. 


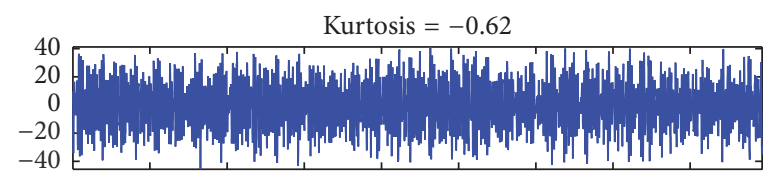

(a)

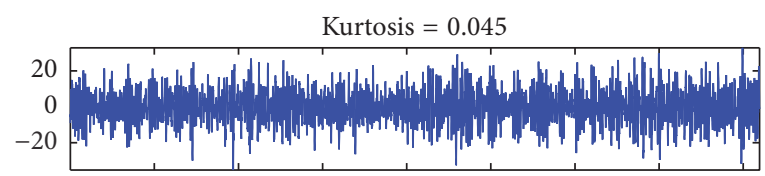

(b)

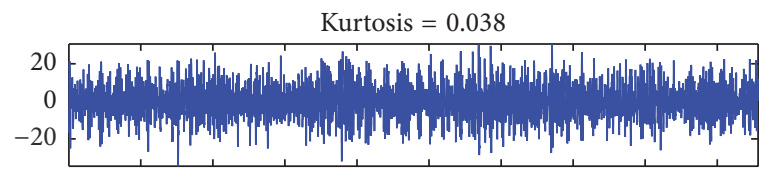

(c)

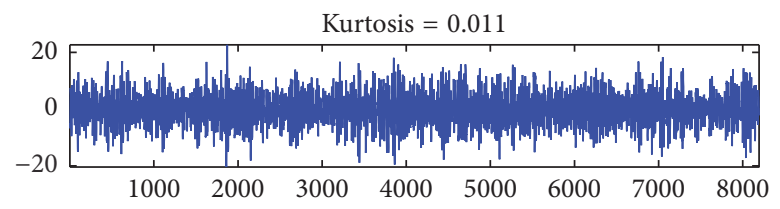

(d)

FIGURE 11: (a) Raw signal [order tracked]. (b) Harmonics of input shaft removed. (c) Harmonics of intermediate shaft removed. (d) Harmonics of output shaft removed.

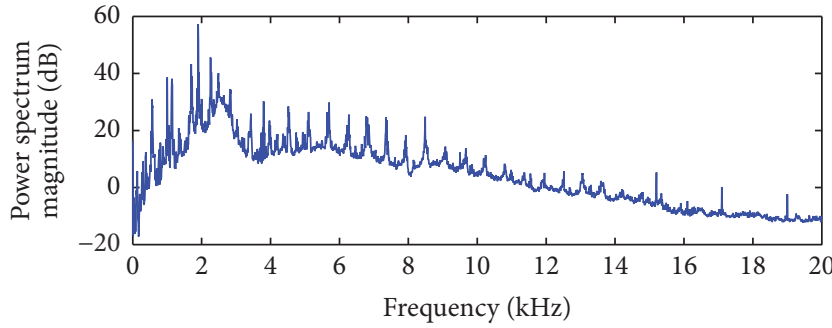

(a)

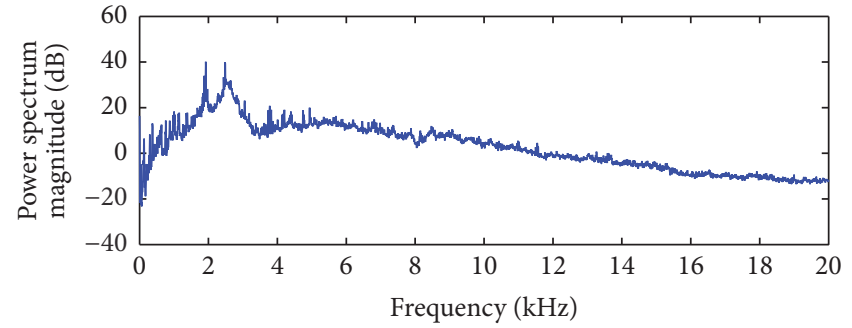

(b)

FIGURE 12: Power spectrum of (a) order-tracked signal and (b) signal processed using the new algorithm.

The end result of steps (1)-(10) is illustrated in Figure 11, which shows the time domain signals (one complete rotation of the output shaft) for each step of removing the shafts' harmonics, that is, the input, intermediate, and output shafts.

Note the disappearance of the discrete components from the spectrum in the processed signal as illustrated in Figure 12.

Figure 13 shows how the new processing algorithm compares between the synchronous averaging approach and the DRS algorithm. Note that the synchronous averaging algorithm on one hand only removed harmonics related to the input shaft. DRS on the other hand gives better results in terms of the removal of all discrete frequencies but disrupts the power spectrum by introducing holes instead of the discrete components.

\section{Summary and Conclusions}

This paper has compared the effect of changing the number of planets of Bell 206 helicopter planetary gearbox on the modulation sidebands around the epicyclic mesh frequency. The planet pass frequency strongly modulates the epicyclic mesh in the 3-planet arrangement. In the case of the 4 planets, the epicyclic mesh frequency and its harmonics are suppressed (other sidebands appear strongly). McFadden and Smith's model [2] was used to predict the highest sidebands/harmonics, which was predicted as 96 and 100 . However, the spectrum and cepstrum showed that modulation in fact happens at two times the carrier frequency $(2 \mathrm{x})$ and thus the highest harmonics were $98 \mathrm{x}$ and $100 \mathrm{x}$. This has been attributed to the idea that planet gears come in pairs of spaced 180 degrees apart with the angle between the 


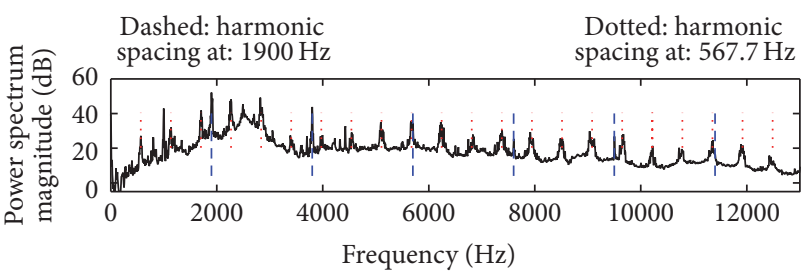

(a)

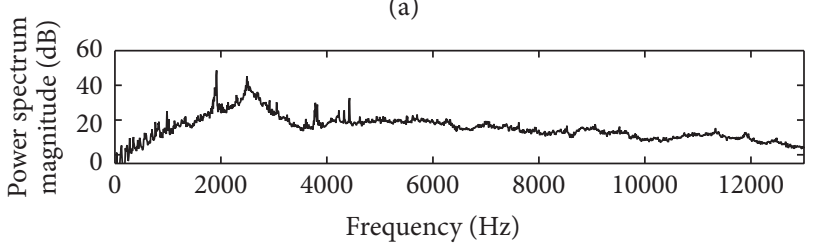

(b)

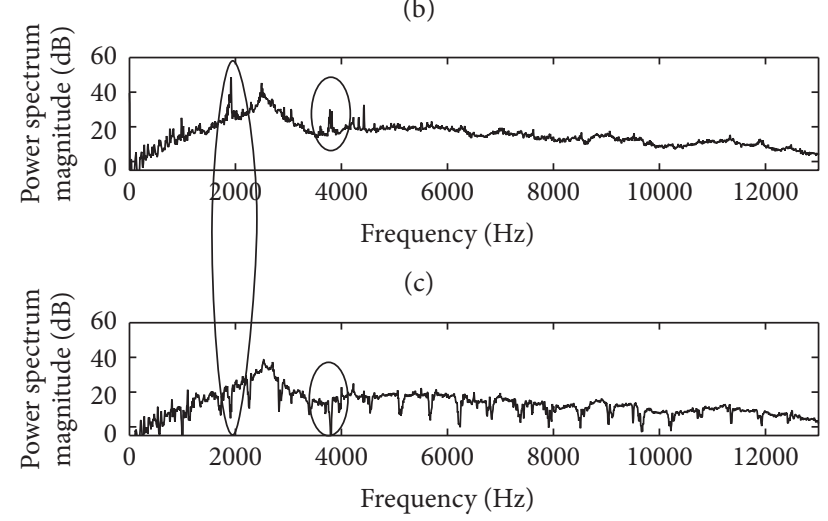

(d)

FIGURE 13: Power spectrum of (a) order-tracked signal, (b) residual signal obtained by setting the shaft' related harmonics to the mean of adjacent (noise) lines, (c) residual signal obtained by subtracting the synchronous averages after resampling, and (d) DRS residual (removing discrete components using DRS).

pairs other than 90 degrees. For the three-planet arrangement, the epicyclic mesh frequency and its harmonics are not suppressed; thus the epicyclic mesh frequency has the highest amplitude with sidebands around it. For the fourplanet arrangement, the epicyclic mesh frequency and its harmonics are suppressed (other sidebands appear strongly). A synchronous/nonsynchronous separation algorithm has been presented to enable the extraction and then removal of the harmonics of the different shafts in the gearbox using only one input tachometer. The algorithm which relies on successive resampling and signal truncation according to the number of shafts gives superior result to those obtained from the discrete random separation algorithm.

\section{Competing Interests}

The author declares that there is no conflict of interests regarding the publication of this paper.

\section{Acknowledgments}

Data used in this paper was provided by Australian Defence Science and Technology Group (DSTG).

\section{References}

[1] J. Pouradier and M. Trouvé, "An assessment of eurocopter experience in HUMS development and support," in Proceedings of the 57th AHS International Annual Forum, American Helicopter Society, Washington, DC, USA, May 2001.

[2] P. D. McFadden and J. D. Smith, "An explanation for the asymmetry of the modulation sidebands about the tooth meshing frequency in epicyclic gear vibration," Proceedings of the Institution of Mechanical Engineers, vol. 199, no. 1, pp. 65-70, 1985.

[3] D. Lewicki and J. Coy, "Vibration characteristics of OH58a helicopter main rotor transmission," NASA Technical Paper NASA TP-2705/AVSCOM TR 86-C-42, 1987.

[4] P. D. McFadden and I. M. Howard, "The detection of seeded faults in an epicyclic gearbox by signal averaging of the vibration,” Propulsion Report 183, Department of Defence, Aeronautical Research Laboratory, 1999.

[5] E. Huff and I. Tumer, "Using triaxial accelerometer data for vibration monitoring of helicopter gearboxes," Journal of Vibration and Acoustics, vol. 128, no. 4, pp. 120-128, 2003.

[6] P. Dempsey, D. Lewicki, and H. Decker, "Transmission bearing damage detection using decision fusion analysis," NASA Technical Paper NASA/TM-2004-213382, 2004.

[7] G. Lang, "S\&V geometry 101," in Sound and Vibration, pp. 1-12, 1999.

[8] J. Antoni and R. B. Randall, "Unsupervised noise cancellation for vibration signals-part II: a novel frequency-domain algorithm," Mechanical Systems and Signal Processing, vol. 18, no. 1, pp. 103-117, 2004.

[9] D. G. Childers, D. P. Skinner, and R. C. Kemerait, "The cepstrum: a guide to processing," Proceedings of the IEEE, vol. 65, no. 10, pp. 1428-1443, 1977.

[10] C. L. Groover, M. W. Trethewey, K. P. Maynard, and M. S. Lebold, "Removal of order domain content in rotating equipment signals by double resampling," Mechanical Systems and Signal Processing, vol. 19, no. 3, pp. 483-500, 2005. 


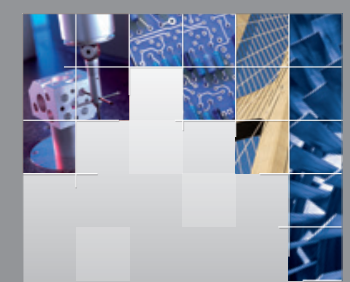

\section{Enfincering}
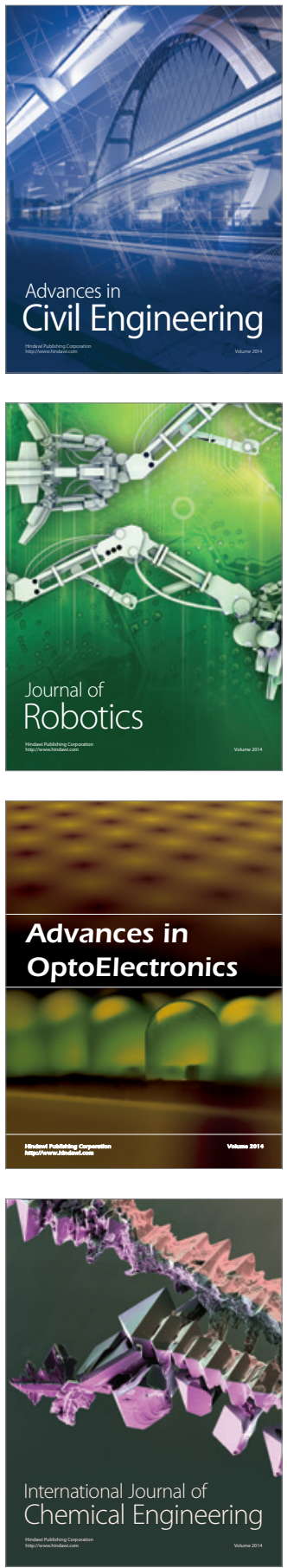

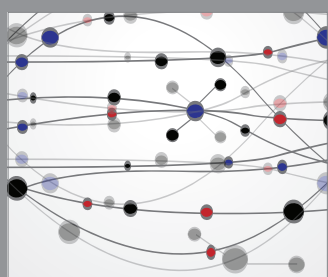

The Scientific World Journal

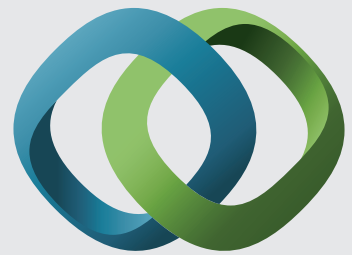

\section{Hindawi}

Submit your manuscripts at

http://www.hindawi.com
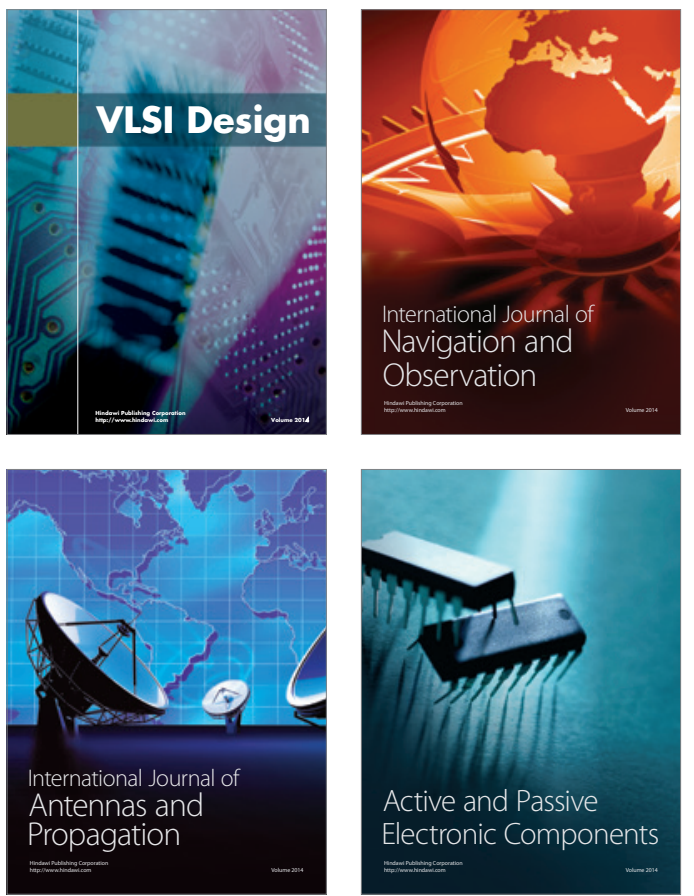
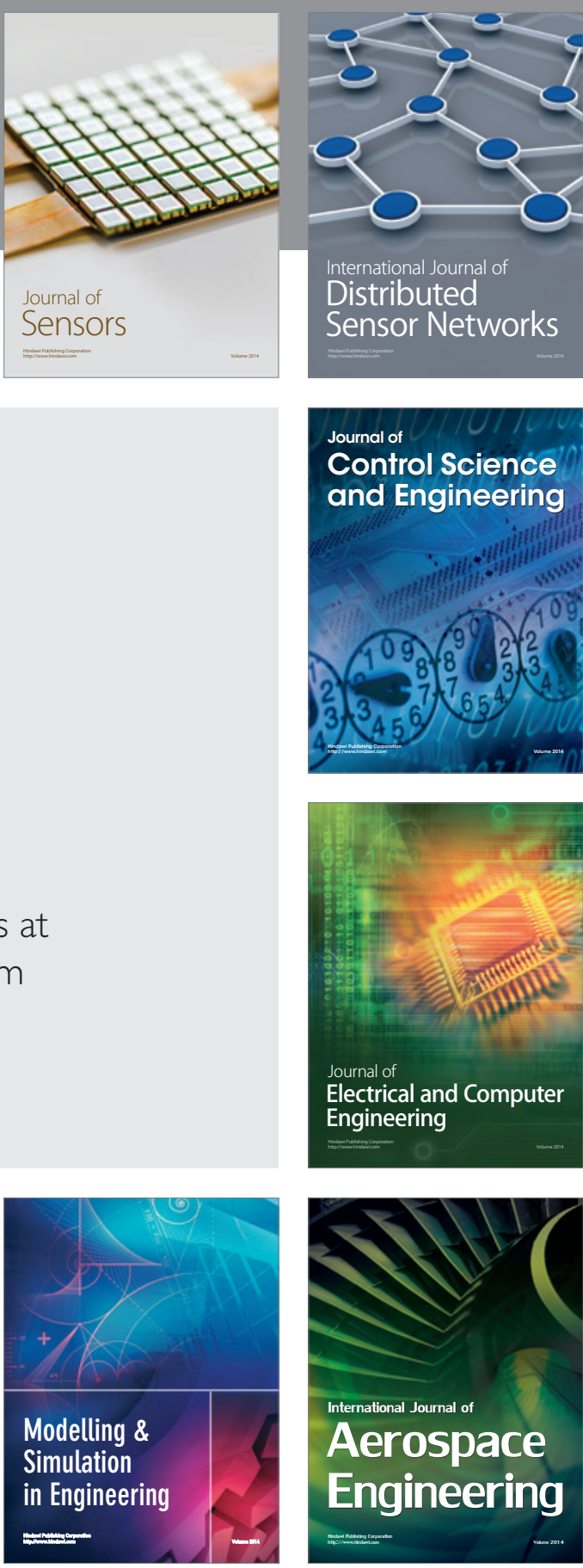

International Journal of

Distributed

Sensor Networks

Journal of

Control Science

and Engineering
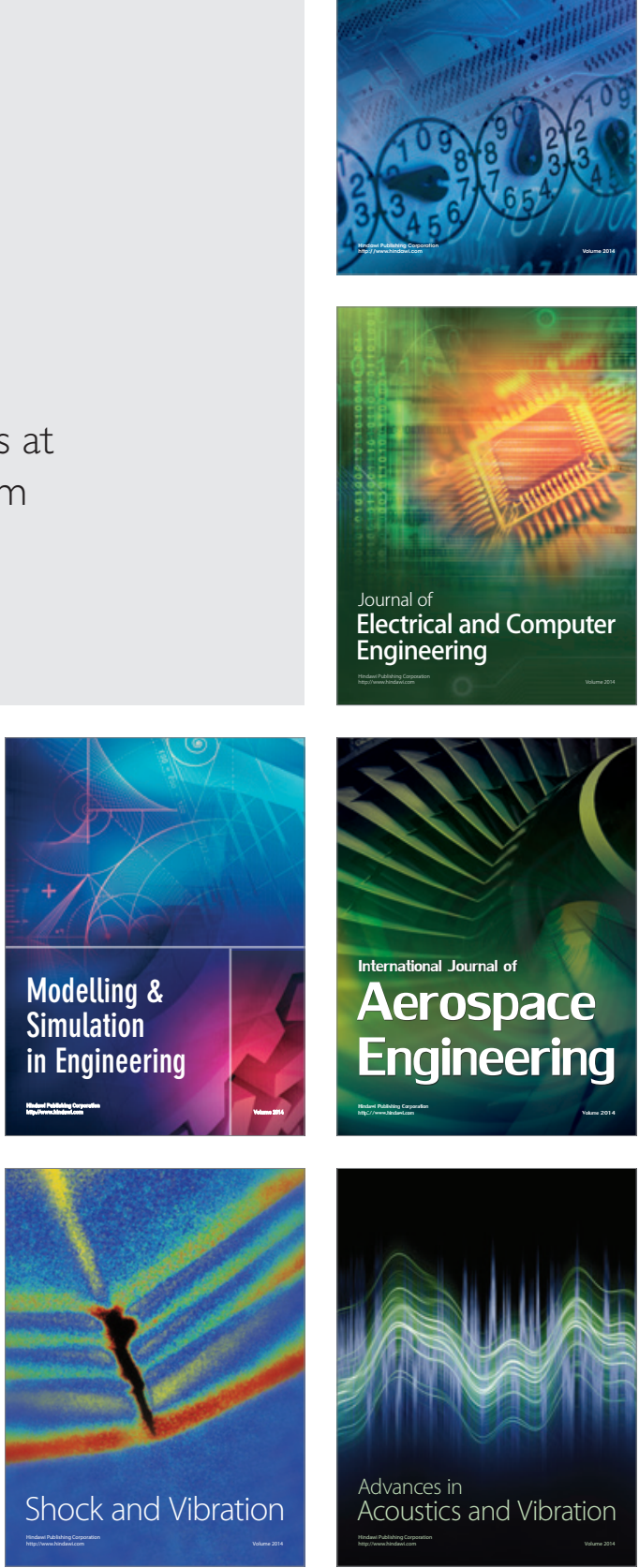